\title{
A rare type 1 hypersensitivity to latex in dental treatment setting and management
}

\author{
Shiva Shankar Bugude \\ Department of Dental and Oral Health, College of Applied Health Sciences in Al Rass, Qassim University, Al Qassim, Saudi Arabia \\ Adv Dermatol Allergol 2022; XXXIX (2): 421-423 \\ DOI: https://doi.org/10.5114/ada.2020.99949
}

Latex gloves are considered to be the most effective barrier to blood-borne pathogens. Latex allergies in health professionals and patients are not quite uncommon. Reactions to latex gloves can vary from minor irritation to allergic reactions. In finished latex products, protein allergens account for up to 2 percent by weight [1]. Latex allergies clinically can manifest as contact dermatitis or immediate type I hypersensitivity or delayed type IV hypersensitivity. This scientific article presents 2 cases of latex allergy type I hypersensitivity and uneventful management.

Case 1. A 28-year-old systemically healthy female patient reported to the dental clinic with a chief complaint of sensitivity in her lower front teeth. Systemic health history was non-contributory. There was no history of any allergy to known agents. The patient has not had any type of dental treatment before. Oral examination revealed malocclusion with generalized calculus deposits on teeth. The patient was educated about her periodontal problems and scheduled for nonsurgical periodontal therapy and provisional splinting of lower anteriors.

During her scheduled visit for periodontal treatment, while performing nonsurgical periodontal therapy in her lower anteriors the patient developed swelling of the lower lip. Swelling was noted within 15-20 min of treatment (Figure 1). Nonsurgical therapy was started without any use of local anaesthesia. Lower lip swelling was not associated with pain or ulceration. The lip colour remained normal. While treating the lower anterior teeth, there was contact of the lower lip with the operator's gloved hand. It was necessary to retract the lower lip for periodontal instrumentation. Considering the possibility of allergic reaction to latex gloves, dental treatment was immediately stopped and further gloves contact was totally avoided. The patient was explained about the situation with her lip. The patient was monitored for her vital signs and emergency medical assistance was called.

Case 2. A 38-year-old systemically healthy female patient visited the dental office for dental check-up to undergo a scaling procedure. It was the patient's first visit to a dental office. Her medical history and drug history were non-contributory. Oral examination revealed relatively healthy teeth and gingiva with moderate supra gingival calculus deposits. The patient was scheduled for ultrasonic oral prophylaxis.

On the day of appointment, when treatment started, the patient gradually developed lower lip swelling within 10 to 15 min (Figure 2). Lower lip swelling was not associated with pain or ulceration. The lip colour remained normal. Oral prophylaxis was performed without any use of local anaesthetic agents. Thus, drug allergy and adverse drug reactions were ruled out. As prophylaxis started with lower anterior teeth, the operator used his gloved hand finger to retract the lower lip for accessibility and visibility for instrumentation. The possible allergy to the latex gloves was considered and the dental treatment was immediately stopped. Further contacts with latex gloves was totally avoided. Medical assistance was immediately called taking into account further worsening of the clinical situation.

Medical management of case 1 and case 2. As both patients were not given any local anaesthesia or preoperative medications, adverse drug reactions were ruled out. Both patients' vital signs were normal. The patients were shifted to emergency medical care. The patients were kept under immediate supervision for the possibility of posterior spread of swelling of the lip into the floor of the mouth and any further consequences. However, swelling was limited to the lower lip only and did not progress further. The patients were immediately given $8 \mathrm{mg}$ antihistamine (Chlorpheniramine) oral dose and $10 \mathrm{mg}$ methylprednisolone oral dose. There were no significant changes in the vital signs. After monitoring the patients for the next $24 \mathrm{~h}$, they were discharged home. Oral antihistamine (Chlorpheniramine) and $8 \mathrm{mg}$ and $10 \mathrm{mg}$ oral methylprednisolone twice daily were prescribed for 3 days. Patients were strictly advised to seek

Address for correspondence: Dr. Bugude Shiva Shankar, Department of Dental Hygiene, College of Applied Health Sciences, Qassim University, Al Qassim region, 51921 Saudi Arabia, phone: +96 6531476028, e-mail: S.ggg@qu.edu.sa, periopal@gmail.com Received: 27.07.2020, accepted: 25.08.2020. 

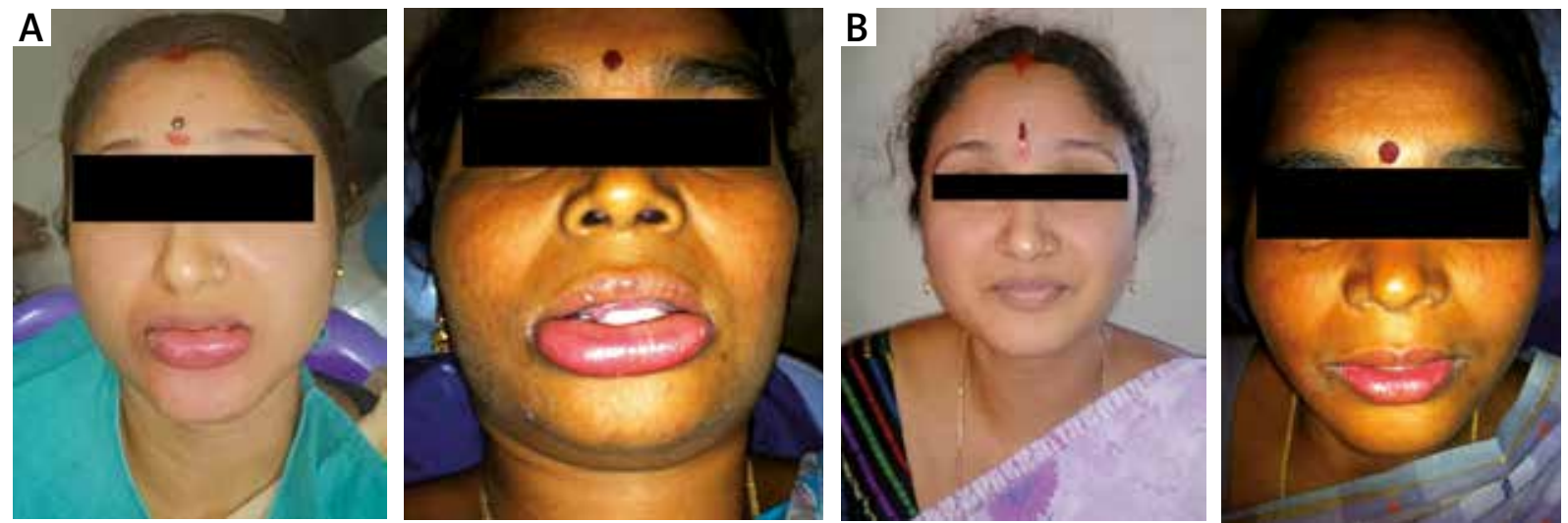

Figure 1. A - Picture showing lip swelling because of type 1 hypersensitivity to latex gloves. B - Total recovery picture after 3 days of taking medications

medical advice in case of any further problems. In the subsequent follow-up period after 3 days, lip swelling was totally reduced in both cases, returning to normal (Figure 1). The patients were educated about the latex allergy and further precautions. Oral antihistamine was stopped and oral hydrocortisone was gradually reduced in tapering doses in the next 2 weeks. Further periodontal treatment was performed wearing non-latex gloves. No untoward events were noted because of the use of latex-free gloves.

The prevalence estimation of latex allergy in the general population is $1 \%$. Latex allergy clinically can manifest as non-allergic contact dermatitis or immediate type I hypersensitivity or delayed type IV hypersensitivity [2]. Non-allergic contact dermatitis and elayed type IV hypersensitivity are the commonest types of latex allergy noted in dental clinical practice.

Contact dermatitis is an immediate response and it is clinically limited to the latex contact area. Clinically, the contact area has presentations such as skin/mucous membrane erythema, vesicles, and chapping [3].

Type IV hypersensitivity occurs 24 to $96 \mathrm{~h}$ following exposure to latex with symptoms like erythema, papules, eczema, weeping, pruritus, and vesicles. Symptoms may or may not expand beyond the area of direct contact [4]. Type IV hypersensitivity is diagnosed by patch testing $[5,6]$.

Type I latex hypersensitivity is less prevalent than the other types. The clinical changes are noted within minutes to a maximum of $2 \mathrm{~h}$ after exposure to the allergen. Symptoms include pruritus, erythema, oedema, rhinoconjunctivitis, urticaria, dyspnoea, palpitations, dizziness, bronchospasm, vasodilation, gastrointestinal cramping, vomiting, hypotension and even death [7].

The diagnosis is based on clinical judgement and ruling out other possibilities. Four different stages have been identified on the basis of reaction severity [8]: 1) urticaria: localized to the contact area, 2) general- ized urticaria with angioedema, 3) urticaria and mucous membrane symptoms, 4) anaphylactic shock.

Both cases presented in the article had no history of prior dental treatment. In both cases the treatment started in the lower anterior region and the lower lip was retracted for accessibility and visibility for periodontal instrumentation. Thus, the clinical manifestations were indeed limited to the major contact area, which is the lower lip. The treatments in both patients were performed without any use of local anaesthesia. This ruled out the possible adverse drug reactions to local anaesthesia or related compounds in the respective agent. The patients did not receive any premedication, which suggested no role of any drug in the clinical changes observed in the lower lip. The observed clinical changes in the lower lip were suggestive of type I or immediate hypersensitivity to latex glove contact. As patients were conscious with no respiratory embarrassment and vitals were stable, oral emergency drugs were employed. The patients were constantly monitored for any worsening changes under medical supervision for the next $24 \mathrm{~h}$. By the fourth day, the patients' lip condition returned to normal. This case report has its own limitations. Both cases were diagnosed as type 1 hypersensitivity to latex based on the clinical course only. There were no further diagnostic skin prick tests carried out to confirm the clinical diagnosis. Patients were not willing to undergo the confirmatory patch tests. Patients were afraid to undergo the skin prick test as it may lead to medical emergencies.

The development of latex allergy is associated with the following high risk factors: 1) spina bifida, 2) family history of atopy, 3) atopy, 4) congenital urinary tract anomalies, 5) history of multiple surgeries or catheterizations, 6) gastrointestinal malformations.

A skin prick test is the gold standard test to diagnose latex allergy [9]. Routine testing of patients and healthcare workers may not be practical, but individuals who fall in the high-risk group should be tested. Allergic reac- 
tions in dental settings can be averted by adapting latex avoidance protocols [8-10]. Latex safe carts should be prepared carrying both treatment materials and supplies. Education of dental office personnel and dental assistants regarding latex allergy is strongly recommended.

To summarize, latex allergy reaction in dental settings is a preventable action. It is possible by education of dental personnel and assistants. The use of non-latex alternatives is highly recommended. It is the obligation of the dentist to identify the high-risk groups and to take appropriate precautions for successful and uneventful outcomes. Being prepared to handle emergency allergic reactions because of human errors is mandatory.

\section{Acknowledgments}

Researchers would like to thank the Deanship of Scientific Research, Qassim University for funding publication of this project.

\section{Conflict of interest}

The author declares no conflict of interest.

\section{References}

1. Minale P, Bignardi D, Troise C, et al. A regional approach to unmet needs in anaphylaxis. Eur Ann Allergy Clin Immunol 2016; 48: 88-93.

2. Shojaei AR, Haas DA. Local anesthetic cartridges and latex allergy: a literature review. J Can Dent Assoc 2002; 68: 622-6.

3. Spina A, Levine HJ. Latex allergy: a review for the dental professional. Oral Surg Oral Med Oral Pathol Oral Radiol Endod 1999; 87: 5-11.

4. Amin A, Palenik CJ, Cheung SW, Burke FJ. Latex exposure and allergy: a survey of general dental practitioners and dental students. Int Dent J 1998; 48: 77-83.

5. Kahn SL, Podjasek JO, Dimitropoulos VA, Brown CW Jr. Natural rubber latex allergy. Dis Mon 2016; 62: 5-17.

6. Warner L. Latex allergy: policy, procedure, and latex-safe box. J Emerg Nurs 1997; 23: 139-41.

7. Nainar SM. Dental management of children with latex allergy. Int J Paediatr Dent 2001; 11: 322-6.

8. Hook D, Fishelberg G. The use of gutta-percha for obturation during root canal therapy in latex-allergic patients. Gen Dent 2003; 51: 337-9.

9. de Groot, Anton C. MD, PhD. New Contact Allergens: 2008 to 2015. Dermatitis 2015; 26: 199-215.

10. Günther KP, Nelitz M, Parsch K, Puhl W. Allergic reactions to latex in myelodysplasia: a review of the literature. J Pediatr Orthop B 2000; 9: 180-4. 